\title{
Investigation of Microdevice Performance by Transient Heat Transfer Simulation
}

\author{
A. Saboonchi and J. Ghasemzadeh \\ Department of Mechanical Engineering, Isfahan University of Technology, Isfahan, 84156-83111, Iran \\ Email: ghasemzadeh@me.iut.ac.ir
}

(Received February 9, 2008; accepted February 16, 2009)

\begin{abstract}
The present work considers transient electrothermal simulation of sub-micrometer silicon device and electron-phonon interactions in electrical and thermal fields. A coupled thermal and electrical model is developed for a silicon $n^{+}-n-n^{+}$structure consisting of the hydrodynamic equations for electron transport and energy conservation equations for phonon. The results indicate that, for one electric field the lattice temperature gradient has significant effect on the magnitude of electric current. The transient phonon temperature affects the device performance due to the change of mobility and gradient temperature of electron. At an external voltage of $0.1 \mathrm{~V}$, calculations show that an increase in the junction boundary temperature by $100{ }^{\circ} \mathrm{C}$, cause increasing the drain current by $16 \%$ at 3 picosecond and decreases it by $17 \%$ up to steady state condition.
\end{abstract}

Keywords: Hydrodynamic, Semiconductor, Heat transfer, Phonon, Electron, Micro scale.

\begin{tabular}{|c|c|c|c|c|c|}
\hline \multicolumn{6}{|c|}{ NOMENCLATURE } \\
\hline$C$ & heat capacity & $J K^{-1} m^{-3}$ & Greeks & & \\
\hline $\mathbf{E}$ & electric fields & $V / m$ & $\varepsilon$ & electric permittivity & $c / V . m$ \\
\hline$e$ & electron charge & $c$ & $k$ & $\begin{array}{l}\text { electron thermal } \\
\text { conductivity }\end{array}$ & $W K^{-1} m^{-1}$ \\
\hline $\mathbf{J}$ & electron current density & $A / m^{2}$ & $\mu$ & electron mobility & $m^{2} / V . s$ \\
\hline$k_{B}$ & Boltzmann constant & $J K^{-1}$ & $\mu_{0}$ & low field mobility & $m^{2} / V . s$ \\
\hline$m^{*}$ & electron effective mass & $k g$ & $v_{s}$ & saturation velocity & $m / s$ \\
\hline$N_{D}$ & $n$-type doping concentration & $m^{-3}$ & $\tau$ & relaxation times & $s$ \\
\hline$n$ & electron number density & $m^{-3}$ & \multicolumn{2}{|l|}{ Subscripts } & \\
\hline $\mathbf{p}$ & electron momentum density & $\mathrm{kg} / \mathrm{m}^{2} . \mathrm{s}$ & $e$ & electron & \\
\hline $\mathbf{Q}$ & heat flow vector & $W / m^{2}$ & $c$ & collision term & \\
\hline$T$ & temperature & $K$ & op & optical & \\
\hline$t$ & time & $s$ & $a$ & acoustic & \\
\hline$V$ & electrostatic potential & $V$ & $m$ & electron momentum & \\
\hline $\mathbf{v}$ & electron drift velocity & $m / s$ & $p h$ & phonon & \\
\hline $\mathbf{W}$ & energy density & $j / m^{3}$ & & & \\
\hline
\end{tabular}

\section{INTRODUCTION}

Increase of the device density on microelectronic chips could be constrained by the ability to dissipate the extremely high rates of heat generation. Thus, it is necessary to understand the mechanisms of heat generation and dissipation inside the device as well as

their effects on electrical performance and reliability. The volumetric heat generation rate inside such a small semiconductor device can be expected to be very high since the device operating power cannot be reduced below a certain level. The energy gained by charge carriers due to an externally applied electrical field, can 
be transferred to the lattice. In equilibrium, the electrons and lattice exhibit similar energy levels. Yet, power device operation requires the application of large fields and high currents. Therefore, these devices normally operate in electrical nonequilibrium. Thermal nonequilibrium refers to the condition when charge carriers are not able to transfer their excess energy to the lattice efficiently. A temperature difference is created between electrons and the lattice resulting in localized heating in the active area of the device. At small scales, thermal transport in semiconductors is usually described in terms of quantized lattice vibrations called phonons.

Numerical simulation of the transient electrical performance of silicon semiconductor devices such as current-voltage characteristics of semiconductor devices has been studied. Early numerical simulation programs referred to Selberherr (1984) used the classic drift-diffusion equation for electron transport. Since the typical applied bias is of the order of $1 \mathrm{~V}$ and the device size has decreased to submicro scales, high electric fields are produced in the device. The energy which electrons gain from the electric field cannot be sufficiently removed by lattice during electron-lattice collision. Therefore, a nonequilibrium situation occurs resulting in the so-called hot electron transport. Blotekjaer (1970) proposed a method to describe hot electron transport, which is now well known as the hydrodynamic model. Numerical simulation results of electron transport have been carried out assuming the lattice to be an isothermal reservoir (Meinerzhagen and Engl 1988; Chai et al. 1992). An electron vorticity equation is also derived and used to investigate many aspects of electron flow structures by Mohseni and Shakouri (2005).

Rangel and Rodriguez (2007) extend the hydrodynamic model of the Boltzmann equation by taking into account the spin of the nonequilibrium carriers injected into semiconducting systems. This spin-resolved hydrodynamic description goes beyond the usual driftdiffusion type approaches in a way that the temporal derivatives of the current densities are considered. This allows us to investigate the transient dynamics of spinpolarized packets in the diffusive and ballistic transport regimes. The dc behavior of single-gate and doublegate MOSFETs with gate lengths ranging from 5 to $100 \mathrm{~nm}$ is simulated using drift-diffusion, hydrodynamic, and Monte Carlo approaches. It is shown that by simple adjustments of the drift-diffusion and hydrodynamic transport model parameters the Monte Carlo currents can be reproduced in the entire gate length range referred to Granzner et al. (2006). This does not consider the heat generation or energy exchange between the lattice and the electrons and therefore cannot be properly used to study lattice thermal effects on electron transport. Since the energy removal rate from the electrons is restricted by the lattice energy, a lattice energy balance must be added to the hydrodynamic model.
Wang (1985) has developed a model including the lattice energy transport equation. Roberts and Chamberlain (1990) have also suggested that the lattice temperature equation must be included in the hydrodynamic equation. A similar model with numerical results was obtained by Katayama and Toyabe (1989). However, none of the articles carefully considered the phonon dispersion relations, which results in different phonon modes. In order to get physical insight into the thermal effects on the electron transport, the interaction of electrons with different phonons at different bias conditions needs to be studied.

A similar model including both the lattice and electron transport was developed for sub-micron GaAs devices by Fushinobu et al. (1995). The thermal and electrical characteristics of sub-micrometer silicon semiconductor devices have studied by considering the nonequilibrium nature of hot electron, optical phonon, and acoustic phonon for steady state conditions by Lai and Majumdar (1996). Numerical simulations were presented of in detail for the transient simulation of the highly coupled non-linear partial differential equations of the full electrons hydrodynamic model (Romano and Russo 2000; Aste and Rudiger 2003) but these results of electron transport have been carried out assuming the lattice to be an isothermal reservoir. The thermal nonequilibrium was determined from phonon temperature distributions obtained using a common electronic solution and three different heating models (Joule heating, electron/lattice scattering, phonon scattering) by Raman et al. (2003).

In this work, 2-D simulations were performed in a partially coupled manner to analyze the electrical and thermal characteristics of the devices. The commercial simulator (ATLAS) was used to perform the electrical characterization. Results from the electrical analysis, such as electron concentrations and electron temperatures, were imported into an in-house semiconductor heating model. Therefore, the results of the electronic solution were used as thermal source terms in the thermal solution. The results of the thermal solution are not coupled back to the electrical analysis. The objective of this article is to concurrently transient study both the thermal and electrical characteristics of sub-micrometer silicon semiconductor devices by considering the nonequilibrium nature of hot electron and phonon, also their interactions in electrical and thermal fields. In the following sections, a coupled thermal and electrical model is developed consisting of the hydrodynamic equations for electron transport and energy conservation equations for phonon.

\section{GOVERNING EQUATIONS}

At low and moderate electric fields, $|\mathbf{E}|<10^{6} \mathrm{~V} / \mathrm{m}$, the electrons mainly interact with acoustic phonons because not many electrons have sufficient energy to excite optical phonons. The ensemble electron drift velocity $\mathbf{v}$ linearly increases with the electric field $\mathbf{E}$, i.e., $\mathbf{v}=\mu \mathbf{E}$, where $\mu$ is the electron mobility. 
This electron transport regime follows Ohms law. However, under a high electric field, $|\mathbf{E}| \geq 10^{6} \mathrm{~V} / \mathrm{m}$, the electrons become energetic enough to interact with optical phonons. In addition, the electron and optical phonon interaction rate can be high enough to saturate the electron drift velocity referred to Wang (1989). Since the electrons interact with the phonons during their transport, the electron mobility is a function of both the electron and the lattice energies referred to Sze (1981)

The electron current density varies as $\mathbf{J}=-e n \mathbf{v}$, where velocity $\mathbf{v}$ is proportional to the mobility for a given electric field. So, we can expect the lattice temperature to influence the device current and therefore the electrical characteristics. It is therefore clear that the semiconductor device characteristics should be determined by the coupled electrical and thermal behavior. A rigorous device simulation must use a concurrent electrical and thermal model that takes into account the lattice heating effect. It is clear that in a nonequilibrium situation, two sub-systems of electrons, phonons, need to be considered in silicon semiconductor devices. The Monte Carlo method is an elegant approach to describe charge transport in semiconductors referred to Jacoboni and Reggiani (1983). However, when phonons have to be incorporated, it is too time consuming and uneconomical to be used for engineering design and analysis of devices.

The hydrodynamic equation is a good engineeringoriented approach to simulate the sub-micron semiconductor devices as long as meaningful expressions of electron transport in terms of average electron velocity and effective electron temperature are valid. The basic equations can be derived from the Boltzmann transport equation, namely the zeroth, first and second-order moments, which represent the electron charge, momentum, and energy conservation, respectively referred to Rudan and Odeh (1986). They are

$$
\begin{aligned}
& \frac{\partial n}{\partial t}+\nabla \cdot(n \mathbf{v})=\left(\frac{\partial n}{\partial t}\right)_{c} \\
& \frac{\partial \mathbf{p}}{\partial t}+\nabla \cdot(\mathbf{v p})=-e n \mathbf{E}-\nabla\left(n k_{B} T_{e}\right)+\left(\frac{\partial \mathbf{p}}{\partial t}\right)_{c} \\
& \frac{\partial \mathbf{W}_{e}}{\partial t}+\nabla \cdot\left(\mathbf{v} \mathbf{W}_{e}\right)=-e n \mathbf{v} \cdot \mathbf{E} \\
& -\nabla \cdot\left(\mathbf{v} n k_{B} T_{e}\right)-\nabla \cdot \mathbf{Q}_{e}+\left(\frac{\partial \mathbf{W}_{e}}{\partial t}\right)_{c}
\end{aligned}
$$

where $\mathrm{n}$ is the electron number density, $\mathbf{v}$ is electron drift velocity, $\mathbf{E}$ is electrical field, and $T_{e}$ is electron temperature. The electron momentum density $\mathbf{p}$ and energy density $\mathbf{W}_{e}$ can be written as $\mathbf{p}=m^{*} n \mathbf{v}$ and $\mathbf{W}_{e}=1 / 2\left(3 n k_{B} T_{e}+m^{*} n \mathbf{v}^{2}\right)$, respectively. All the last terms with subscript $c$ in the above equations represent the quantities changing due to collision referred to Schlichting (1979). In the electron momentum conservation equation, the driving forces are the electric field and electron number density as well as temperature gradients, whereas the drag force is electron-phonon collision. The heat flow vector $\mathbf{Q}_{e}$ can be found from higher order moments of the Boltzmann transport equation. The Fourier law was suggested to get a better approximation. Therefore,

$$
\mathbf{Q}_{e}=-k_{e} \nabla T_{e}
$$

Where $k_{e}$ is the electron thermal conductivity. The energy conservation equations for optical and acoustic phonons are

$$
\begin{aligned}
\frac{\partial \mathbf{W}_{o p}}{\partial t} & =\left(\frac{\partial \mathbf{W}_{e}}{\partial t}\right)_{c}+\left(\frac{\partial \mathbf{W}_{o p}}{\partial t}\right)_{c} \\
\frac{\partial \mathbf{W}_{a}}{\partial t} & =\nabla \cdot\left(k_{a} \nabla T_{a}\right)-\left(\frac{\partial \mathbf{W}_{o p}}{\partial t}\right)_{c}
\end{aligned}
$$

Where $\mathbf{W}_{o p}$ and $\mathbf{W}_{a}$ are optical and acoustic phonon energy densities, respectively, each of which is related to the heat capacity $C$ as $d \mathbf{W}=C d T$, and $k_{a}$ is the lattice thermal conductivity. The heat capacities of optical phonons $C_{\text {op }}$ and acoustic phonons $C_{\text {a }}$ can be estimated from the Einstein and Debye models, respectively referred to Wang (1989). When $|\mathbf{E}|<10^{6} \mathrm{~V} / \mathrm{m}$, the electrons lose energy directly to acoustic phonons as expressed by

$$
\frac{\partial \mathbf{W}_{a}}{\partial t}=\nabla \cdot\left(k_{a} \nabla T_{a}\right)-\left(\frac{\partial \mathbf{W}_{e}}{\partial t}\right)_{c}
$$

Referred to Arora et al. (1982), all the collision terms can be phenomenologically expressed using the relaxation time approximation as

$$
\begin{aligned}
& \left(\frac{\partial \mathbf{p}}{\partial t}\right)_{c}=-\frac{n m^{*} \mathbf{v}}{\tau_{m}} \\
& \left(\frac{\partial \mathbf{W}_{e}}{\partial t}\right)_{c}=-n \frac{\frac{3}{2} k_{B} T_{e}+\frac{1}{2} m^{*} \mathbf{v}^{2}-\frac{3}{2} k_{B} T_{p h}}{\tau_{e-p h}} \\
& \left(\frac{\partial \mathbf{W}_{o p}}{\partial t}\right)_{c}=-C_{p h} \frac{T_{o p}-T_{a}}{\tau_{o p-a}}
\end{aligned}
$$

where $\tau_{m}, \tau_{e-p h}$, and $\tau_{o p-a}$ are relaxation times for electron momentum, electron energy, and optical phonon, respectively, $T_{p h}$ can be either optical or acoustic phonon temperature depending on which kind of phonons the electrons interact with. The above analysis can also be applied to the holes in Si. However, in the active region of a semiconductor device, either the electrons or the holes are the majority carrier. The current contributed by the minority carriers is much smaller than that contributed by the majority carriers. Therefore, the minority carriers can be simply assumed to follow the drift diffusion model. For example, holes are minority carriers in a $n$-channel MOSFET. MOSFET stands for metal oxide semiconductor field effect transistor. 
Therefore, to solve for the electric field, the Poisson equation is used and is given as

$$
\nabla \cdot(\varepsilon \mathbf{E})=e\left(N_{D}-n\right)
$$

With

$$
\mathbf{E}=-\nabla V
$$

Where $\mathrm{V}$ electrostatic potential, $\varepsilon$ is the permittivity which is different for silicon and silicon dioxide, $N_{D}$ is the $n$-type doping concentration. Finally, Eqs. (1)-(4) and (5)-(11) form a closed system.

In our simulations for silicon, we will use the Baccarani and Wordemann (1985) model, which defines the relaxation times by

$$
\begin{aligned}
& \tau_{m}=\frac{m^{*} \mu}{e} \\
& \tau_{e-p h}=\tau_{m}\left(\frac{1}{2}+\frac{3 k_{B}}{2 m^{*} v_{s}^{2}} \frac{T_{e}^{2}}{T_{e}+T_{p h}}\right)
\end{aligned}
$$

$v_{s}$ is the saturation velocity, i.e. the drift velocity of the electron gas at high electric fields.

According to (Sze 1981; Singh 1993; Ng 1995 and Caughey 1967) the low field mobility is given by the empirical formula for silicon, which depends mainly on the lattice temperature and the total doping density

$$
\begin{aligned}
& \mu_{0}\left(N_{D}, T_{p h}\right)=80\left(\frac{T_{p h}}{300}\right)^{-0.45}+ \\
& 1430\left(\frac{T_{p h}}{300}\right)^{-2}-80\left(\frac{T_{p h}}{300}\right)^{-0.45} \\
& 1+\left(\frac{N_{D}}{1.12 \times 10^{17}\left(\frac{T_{p h}}{300}\right)^{3.2}}\right)^{0.72\left(\frac{T_{p h}}{300}\right)^{0.065}}
\end{aligned}
$$

and the electron mobility is given by

$$
\mu\left(E, N_{D}, T_{p h}\right)=\frac{\mu_{0}}{\sqrt{1+\left(\mu_{0} E / v_{s}\right)^{2}}}
$$

This is the well-known Caughey and Thomas (1967) mobility model. Blotekjaer (1970) and Baccarani and Wordemann (1985) (BBW) proposed a model for heat flow vector $\mathbf{Q}_{e}$ that its thermal conductivity descript with Eq. (17).

$$
k_{e}=\left(\frac{5}{2}+r\right) n \frac{k_{B}^{2} \mu}{e} T_{e}
$$

Several different choices for $\mathrm{r}$ can be found in the literature, and many authors such as (Feng and Hintz 1988; Alsunaidi et al. 1997).

According to Gnudi et al. (1990) with comparisons of hydrodynamic and MC simulations of the ballistic diode, the best value for $r$ appears to be -2.1 for silicon at $300 \mathrm{~K}$.

According to $\mathrm{Ng}$ (1995) lattice thermal conductivity descript with Eq. (18).

$k_{a}=148\left(\frac{T_{p h}}{300}\right)^{-1.65}$

These relations are valid between 300 and $800 \mathrm{~K}$ for silicon.

The above system of nonlinear equations can be simplified. According to Cook and Fery (1982) first, the convective inertia term $\nabla .(\mathbf{v p})$ in the electron momentum conservation equation can be neglected because this term is small compared to the other driving forces or the collision term and $T_{e} \nabla n$ is small compared to $n \nabla T_{e}$ for this case. Finally, the momentum equation can be written as

$$
\mathbf{J}-e n \mu m^{*} \frac{\partial \mathbf{v}}{\partial t} \approx-e n \mu\left(\nabla V-\frac{k_{B}}{e} \nabla T_{e}\right)
$$

Eq. (19) shown that if voltage term $\nabla V$ to be in order $0.1 \mathrm{~V}$, gradient electron temperature will be have important effect on electron current density.

\section{NUMERICAL METHOD}

Let us write the 1-D version of the equations of the two models:

$$
\begin{aligned}
& \frac{\partial}{\partial t} n+\frac{\partial}{\partial x}(n \mathbf{v})=0 \\
& \frac{\partial}{\partial t} \mathbf{p}+\frac{\partial}{\partial x}\left(\mathbf{v p}+n k_{B} T_{e}\right)=-e n \mathbf{E}-\frac{n m^{*} \mathbf{v}}{\tau_{m}} \\
& \frac{\partial}{\partial t} \mathbf{W}_{e}+\frac{\partial}{\partial x}\left(\mathbf{v} \mathbf{W}_{e}+\mathbf{v} n k_{B} T_{e}+\mathbf{Q}_{e}\right)= \\
& -e n \mathbf{v} \cdot \mathbf{E}-\frac{3}{2} n k_{B}\left(\frac{T_{e}-T_{p h}}{\tau_{e-p h}}\right)-\frac{1}{2} \frac{n m^{*}}{\tau_{e-p h}} \mathbf{v}^{2} \\
& \frac{\partial}{\partial t} \mathbf{W}_{p h}+\frac{\partial}{\partial x} \mathbf{Q}_{p h}=\frac{3}{2} n k_{B}\left(\frac{T_{e}-T_{p h}}{\tau_{e-p h}}\right)
\end{aligned}
$$

In this case, the thermal system is represented as a single lattice temperature and is considered to be in thermal equilibrium for optical and acoustic phonons. The heat flow vector $\mathbf{Q}_{p h}$ can be written as $\mathbf{Q}_{p h}=-k_{p h} \nabla T_{p h}$ and $k_{p h}=k_{a}$ is the lattice thermal conductivity and $\mathbf{W}_{p h}$ can be written as $\mathbf{W}_{p h}=C_{p h} T_{p h}$.The heat capacities of phonons According to $\mathrm{Ng}$ (1995) $C_{p h}$ can be estimated from

$$
C_{p h}=711+255 \frac{\left(\frac{T_{p h}}{300}\right)^{1.85}-1}{\left(\frac{T_{p h}}{300}\right)^{1.85}+\frac{255}{711}}
$$


These relations are valid between 50 and $800 \mathrm{~K}$ for silicon.

We make use of a splitting scheme, based on the following decomposition. Let us consider a system of the form

$$
\frac{\partial u}{\partial t}+\frac{\partial f}{\partial x}=g
$$

We define the following vector fields:

$$
\begin{aligned}
& u=\left(n, \mathbf{p}, \mathbf{W}_{e}, \mathbf{W}_{p h}\right) \\
& f=\left(n \mathbf{v}, \mathbf{v p}+n k_{B} T_{e}, \mathbf{v} \mathbf{W}_{e}+\mathbf{v} n k_{B} T_{e}+\mathbf{Q}_{e}, \mathbf{Q}_{p h}\right) \\
& g=\left(0,-e n \mathbf{E}-\frac{n m^{*} \mathbf{v}}{\tau_{m}},-e n \mathbf{v} \cdot \mathbf{E}-\frac{3}{2} n k_{B}\right. \\
& \left.\left(\frac{T_{e}-T_{p h}}{\tau_{e-p h}}\right)-\frac{1}{2} \frac{n m^{*}}{\tau_{e-p h}} \mathbf{v}^{2}, \frac{3}{2} n k_{B}\left(\frac{T_{e}-T_{p h}}{\tau_{e-p h}}\right)\right)
\end{aligned}
$$

Then, for each time step, a numerical approximation $\tilde{u}$ of the solution is obtained by solving the two consecutive steps:

$$
\begin{array}{ll}
\frac{\partial u_{1}}{\partial t}+\frac{\partial f}{\partial x}=0, & \text { convection step } \\
u_{1}(t)=\tilde{u}(t) & \\
\frac{\partial \tilde{u}}{\partial t}=g, & \text { relaxation step } \\
\tilde{u}(t)=u_{1}(t+\Delta t) &
\end{array}
$$

According to Romano and Russo (2000) the numerical scheme is a splitting scheme based on the Nessyahu and Tadmor (1990) scheme for the hyperbolic step, and a semi-implicit Euler scheme for the relaxation step. Note that this method is second-order accurate in space, but only first order accurate in time.

Our program (HDM) compared with Romano and Russo (2000) results as per Fig. 1.

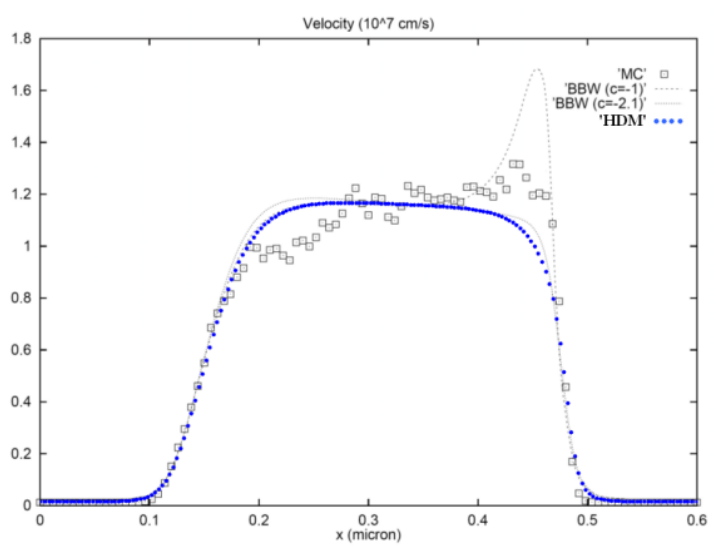

Fig. 1. Velocity profile $\left(\times 10^{7} \mathrm{~cm} / \mathrm{sec}\right)$ of HDM with BBW model with $\mathrm{c}=-1.0$ (dashed line), $\mathrm{c}=-2.1$ (dotted line) and with Monte-Carlo simulation (squares). The channel length is $0.4 \mu \mathrm{m}$, and the applied voltage is $1 \mathrm{~V}$.
At this figure, our results compared with BBW and MC (Mount Carlo Simulation). At Eq. (17), $r$ parameter is c parameter in this figure.

\section{NUMERICAL STUDY}

We simulated a $n^{+}-n-n^{+}$ballistic diode, as shown in Fig. 2., which models the electron flow in the channel of an MOSFET, and exhibits hot electron effects at scales on the order of a micrometer. Our diode begins with a $0.1 \mu m n^{+}$'source' region with doping density $N_{D}=10^{18} \mathrm{~cm}^{-3}$, is followed by a $0.1 \mu \mathrm{m} \mathrm{n}$ 'channel' region $N_{D}=2 \times 10^{15} \mathrm{~cm}^{-3}$, and ends with a $0.1 \mu \mathrm{m} \mathrm{n}^{+}$'drain' region $N_{D}=10^{18} \mathrm{~cm}^{-3}$. The doping density was slightly smeared out at the junctions.

We considered silicon at $T_{p h}=300 \mathrm{~K}$ for initially conditions and solved electrons hydrodynamic equations for this constant lattice temperature until steady state results for electrons as shown in Figs. 4, 5. Then, these results are used in initial conditions as soon as the phonon temperature is set $400 \mathrm{~K}$ in junction boundary $\left(T_{p h-\text { junction }}=400 \mathrm{~K}\right)$ as shown in Fig. 1 .

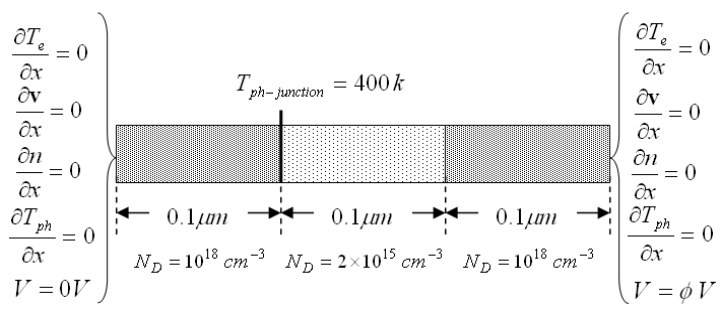

Fig. 2. The microscale simulation regions of a ballistic diode.

This problem solved for $\phi=1$ and $\phi=0.1$.

We can develop this problem for other micro devices as micro thermoelectric cooler and generator and etc. which are content two type of semiconductor in series connections, too.

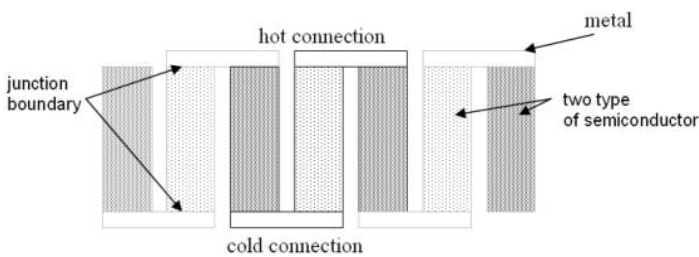

Fig. 3. View of some micro devices as micro thermoelectric cooler and generator.

A fine uniform mesh of $3 \mathrm{~nm}$ is used in the microscale region which guarantees a spacing of less than the Debye length in the device active region and reduces the error due to discretization referred to Sze (1981). 


\section{RESUltS AND DiscuSSIONS}

We have considered silicon at $T_{p h}=300 \mathrm{~K}$ and solved electrons hydrodynamic equations until steady state time. In our simulations, the required time to reach steady state is about five picoseconds. Figure 4 shows the distributions of (a) electron density, (b) electron velocity, (c) lattice temperature and (d) electron temperature that the drain-to-source voltage bias $\mathrm{V}$ is 1 V. For approving the simulation, outputs results were compared with previous published results such as Romano and Russo (2000); Aste and Rudiger (2003). A comparison between numerical results and Monte-Carlo simulations shows that BBW model gives satisfactory results for electron velocity and energy, provided by choosing a suitable value for $r$ in Eq. (17) for a fitting parameter contained in the expression for the heat conductivity. Therefore we considered $r=-2.1$ for this present work.

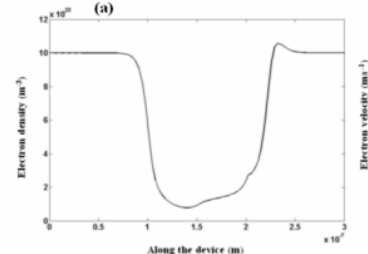

(c)
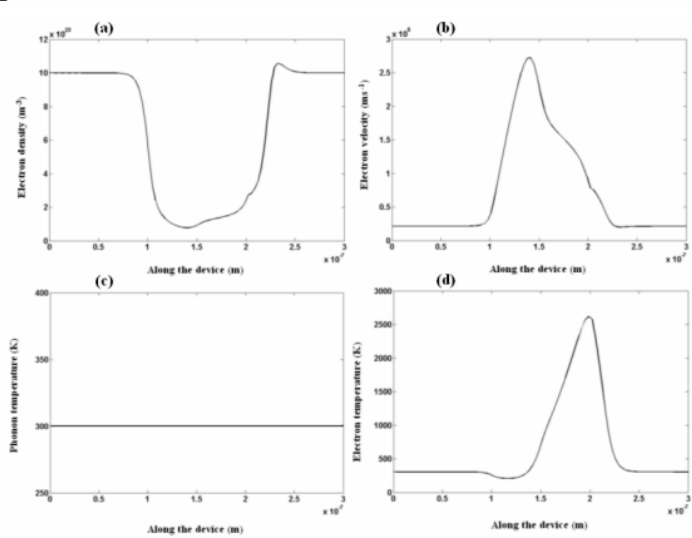

Fig. 4. Shows the distributions of electron density, (b) electron velocity, (c) lattice temperature and (d) electron temperature for voltage bias $1 \mathrm{~V}$ and the lattice temperature $300 \mathrm{~K}$.

When the junction boundary temperature is fixed in 400 $\mathrm{K}$, the lattice temperature develops in microdevice up to 1.8 nanosecond duration as shown in Fig. 5.

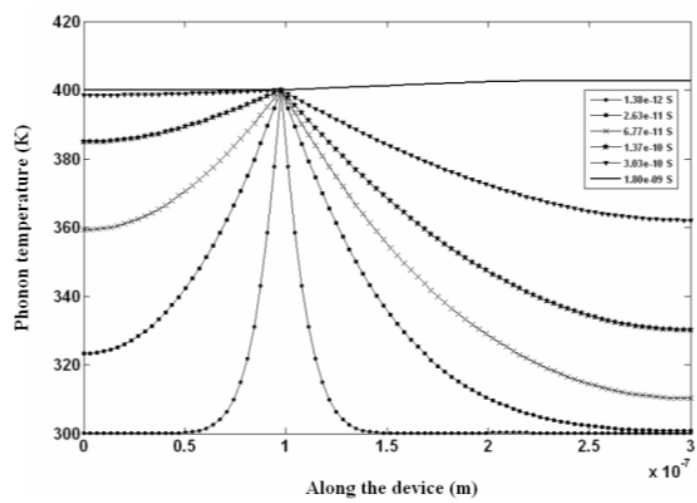

Fig. 5. Shows the distributions of lattice temperature in device along various times.

The constant timestep $\Delta t$ used in our simulations was typically in a few tenths of a femtosecond order, therefore one run of this study has twenty million of iterations approximately up to steady state condition. The temperature rose up $403 \mathrm{~K}$ near the second junction of device because electron temperature has maximum value in this region. The electron temperature is high in the second junction, and the thermal source will have the largest amount in this region for phonon energy equation.

Simultaneously, thermal diffusion occurs and the temperature profiles spread over the entire device. A single MOSFET device is not expected to dissipate enough power to significantly increase lattice temperature. Whereas energy density of electron is five orders lower than energy density of phonon, therefore effects of electron temperature is very weak than phonon conduction term. Hence, although electron temperature reaches to $2700 \mathrm{~K}$ in near the second junction of device but the increase of maximum lattice temperature is $3 \mathrm{~K}$ in this region.

Eq. (19) shows that if drain-to-source voltage bias V to be $1 \mathrm{~V}$, gradient temperature won't have important effect on electron current density. The increase of lattice temperature reduces electron velocity due to the reduction of electron mobility as shown in Fig. 6.

The rate of electron velocity reduction is very high up to 0.3 nanosecond, because the rate of phonons heat transfer is very high up to the stated time.

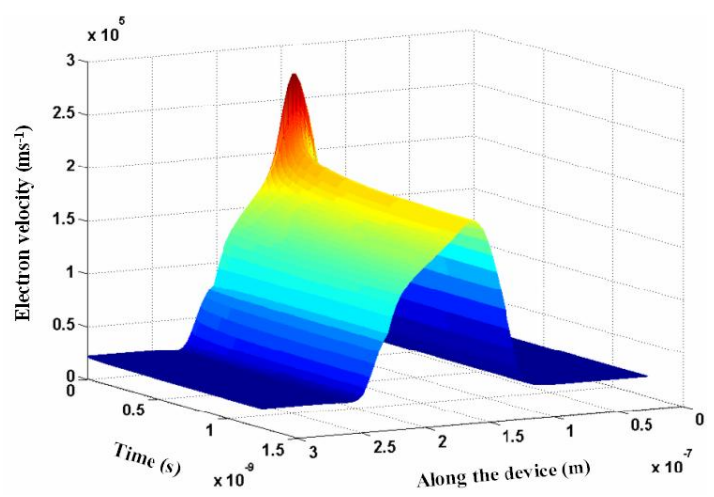

Fig. 6. Shows the contour of velocity in device along various times.

At an external voltage of $1 \mathrm{~V}$, calculations show that an increase in the junction boundary temperature by 100 ${ }^{\circ} \mathrm{C}$ decreases the electron velocity and drain current by $14 \%$ up to steady state time as shown in Fig.7.

The increase of lattice temperature in junction boundary increases electron temperature but decrease of electron velocity will reduces maximum electron temperature. Interactions of these two effects can make increase or decrease of maximum electron temperature according to order of any case magnitude. Therefore this boundary condition increases the maximum electron temperature by $2 \%$ in 10 picoseconds then it reduces by $4 \%$ up steady state time as shown in Fig. 8. However gradient electron temperature had increased but it was very lower than electrical field term in Eq. (19) and it is not practically considered. 


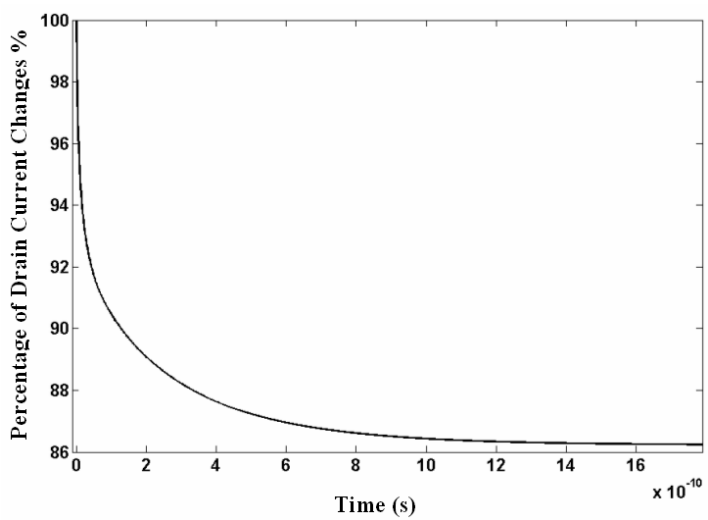

Fig. 7. Shows the variation of Percentage of Drain Current Changes at various times.

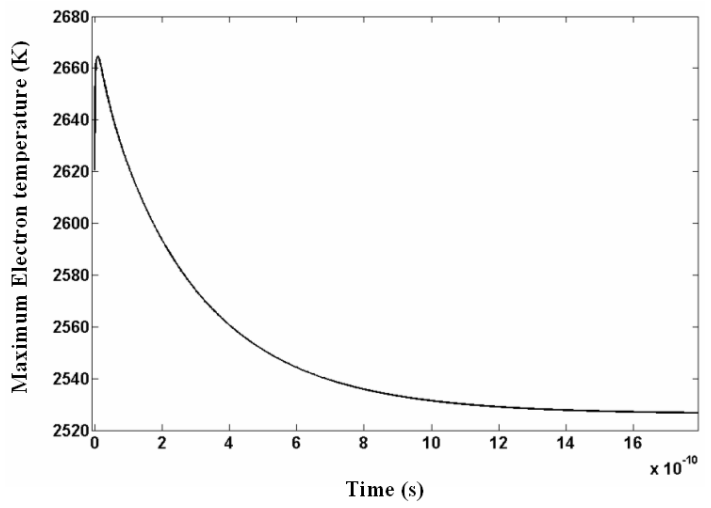

Fig. 8. Shows the variation of maximum electron temperature at various times.

Eq. (19) shows that if drain-to-source voltage bias $\mathrm{V}$ to be $0.1 \mathrm{~V}$, gradient temperature will be have important effect on electron current density. At an external voltage of $0.1 \mathrm{~V}$, calculations show that an increase in the junction boundary temperature by $100{ }^{\circ} \mathrm{C}$, increases the electron current by $17 \%$ in 3 picoseconds then it reduces by $16 \%$ up to steady state time as shown in Fig. 9.

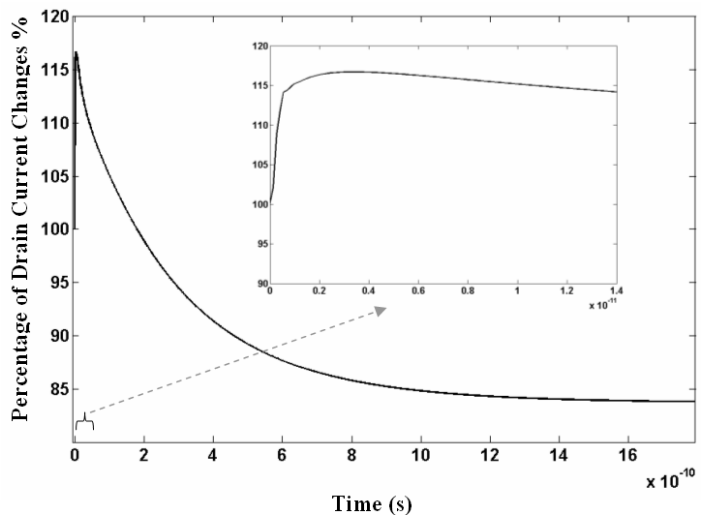

Fig. 9. Shows the variation of Percentage of Drain Current Changes at various times.

The order of gradient electron temperature is greater than electric field in initially times whereas the electron mobility is not seriously decreased yet at this time, therefore the electron current increases up to 3 picoseconds. Then heat transfer reduces gradient temperature and also increase of lattice temperature decreases electron mobility, so the electron current reduces after this time.

The increase of lattice temperature in junction boundary increases electron temperature but decrease of electron velocity will reduce maximum electron temperature. Interactions of these two effects can cause increase or decrease of maximum electron temperature in according to order of any case magnitude. Therefore this boundary condition increases the maximum electron temperature by $80{ }^{\circ} \mathrm{C}(21 \%)$ up to steady state time as shown in Fig. 10.

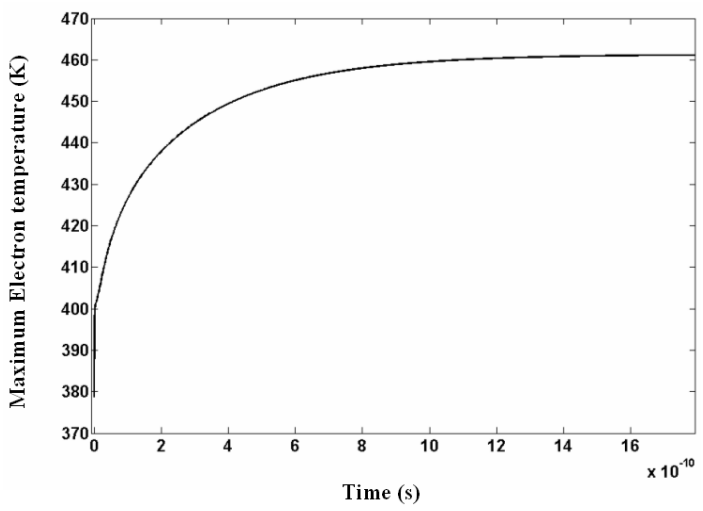

Fig. 10. Shows the variation of maximum electron temperature at various times.

As a fundamental result of this work, transient electrical characteristics of power devices are closely coupled with nonequilibrium thermal effects. The findings lead us to believe that devices with similar or smaller dimensions with short time scale features are susceptible to thermal nonequilibrium effects. This investigation using a fully coupled computational method in transient state has been implemented and determines the transient effect of each model on electrical performance.

\section{CONCLUSION}

Finally, a one-dimensional numerical simulation program in transient state is developed in the microscale region to obtain electrical characteristics and lattice heat transport. The distributions of electron velocity, maximum electron temperature, and lattice temperature are predicted. The transient phonon temperature affects the device performance due to the change of mobility and gradient temperature of electron.

At an external voltage of $1 \mathrm{~V}$, calculations show that an increase in the junction boundary temperature by 100 ${ }^{\circ} \mathrm{C}$ decreases the electron velocity and drain current by $16 \%$ up to steady state time as again it increases the maximum electron temperature by $2 \%$ in 10 picoseconds then it reduces by $3.5 \%$ up steady state time. Also at an external voltage of $0.1 \mathrm{~V}$, calculations show that an increase in the junction boundary temperature by $100{ }^{\circ} \mathrm{C}$, increases the electron current 
by $17 \%$ in 3 picoseconds then it reduces by $16 \%$ up to steady state time as again it increases the maximum electron temperature by $21 \%$.

\section{REFERENCES}

Alsunaidi, MA., S.M. Hammadi and S.M. El-Ghazaly (1997). A parallel implementation of a twodimensional hydrodynamic model for microwave semiconductor device including inertia effects in momentum relaxation. International Journal of Numerical Modelling: Electronic Networks, Devices and Fields 10, 107-119.

Arora, N.D., J.R. Hauser, and D.J. Roulston (1982). IEEE Trans. Electron Devices 29, 292.

Aste, A. and R. Vahldieck (2003). Time-domain simulation of the full hydrodynamic model. International Journal of Numerical Modeling: Electronic Networks, Devices and Fields 16, 161174 .

Baccarani, G., M.R. Wordemann (1985). An investigation of steady-state velocity overshoot in silicon. Solid-State Electronics 28, 407-416.

Blotekjaer, K. (1970). IEEE Trans. Electron. Devices 17,38 .

Caughey, D. and R. Thomas (1967). Carrier Mobilities in Silicon Empirically Related to Doping and Field. Proc. IEEE 52, 2192-2193.

Chai, K.W., P.A. Mawby and A. McCowen (1992). Int. J. Numerical Modeling: Electronic Networks, Devices and Fields 5, 53.

Cook, R.K. and J. Fery (1982). Compel-Int, J. Computer and Mathematics in Electrical and Electronic Engineering 2, 65.

Feng, Y.K. and A. Hintz (1988), Simulation of submicrometer GaAs MESFETs using a full dynamic transport model. IEEE Transactions on Electron Devices 35, 1419-1431.

Fushinobu, K., A. Majumdar and K. Hijikata (1995). J. Heat Transfer 117, 27.

Gnudi, A., F. Odeh and M. Rudan (1990). Investigation of nonlocal transport phenomena in small semiconductor devices. European Transactions on Telecommunications and Related Technologies 1, 307-312.

Granzner, R., V.M. Polyakov, F. Schwierz, M. Kittler, Luyken, R.J., W. Rösner and M. Städele (2006). Simulation of nanoscale MOSFETs using modified drift-diffusion and hydrodynamic models and comparison with Monte Carlo results. Microelectronic Engineering 83(2), 241-246.

Jacoboni, C. and L. Reggiani (1983). Rev. Mod. Phys. 55,645 .
Katayama, K. and T. Toyabe (1989). IEEE IEDM 89, 135.

Lai, J. and A. Majumdar (1996). Concurrent thermal and electrical modeling of sub-micrometer silicon devices. J. Appl. Phys. 79(9), 7353- 7361.

Meinerzhagen, B. and W.L. Engl (1988). IEEE Trans. Electron Devices 35, 689.

Mohseni, K. and A. Shakouri (2005). Electron vortices in semiconductors devices. PHYSICS OF FLUIDS 17, 100602 .

Nessyahu, H. and E. Tadmor (1990). Non-oscillatory central differencing for hyperbolic conservation law. J. Comput. Phys. 87, 408-463.

$\mathrm{Ng}$, K. (1995). Complete Guide to Semiconductor Devices, McGraw-Hill.

Raman, A., D.G. Walker and T.S. Fisher (2003). Simulation of nonequilibrium thermal effects in power LDMOS transistors. Solid-State Electronics.

Rangel-Huerta, A. and M.A. Rodriguez-Meza (2007). Hydrodynamic model for spin-polarized electron transport in semiconductors. J. Appl. Phys. 101(5).

Roberts, J. W. and S.G. Chamberlain (1990). CompelInt. J. Computer and Mathematics in Electrical and Electronic Engineering 9(1).

Romano, V. and G. Russo (2000). Numerical Solution for Hydrodynamical Models of Semiconductors. Mathematical Models and Methods in Applied Sciences 10(7), 1099-1120.

Rudan, M. and F. Odeh (1986). Compel-Int. J. Computer and Mathematics in Electrical and Electronic Engineering 5(149).

Schlichting, H. (1979). Boundary-Layer Theory. 7th ed., McGraw-Hill, New York.

Selberherr, S. (1984). Analysis and Simulation of Semiconductor Devices. Springer, New York.

Singh, J. (1993). Physics of Semiconductors and their Heterostructures. McGraw Hill.

Sze, S.M. (1981). Physics of Semiconductor Devices. 2nd ed. Wiley, New York.

Wang, C.T. (1985). Solid-State Electron 28(783).

Wang, S. (1989). Fundamentals of Semiconductor Theory and Device Physics. Prentice-Hall, Englewood Cliffs, NJ. 